\title{
Drug Treatment of Disease
}

\section{CHLORPROMAZINE AND ALLIED PHENOTHIAZINE DERIVATIVES}

\author{
BY \\ LINFORD REES, M.D., B.Sc., F.R.C.P., D.P.M. \\ Physician-in-Charge of the Department of Psychological Medicine, St. Bartholomew's Hospital, London; \\ Physician, the Bethlem Royal and the Maudsley Hospital, London
}

Chlorpromazine hydrochloride, introduced by Laborit and Huguenard (1951), was found to have a wide range of interesting pharmacological actions and therapeutic applications in medicine, surgery, anaesthesia, and psychiatry. It is a depressant of the central nervous system with comparatively little action on the cerebral cortex, and exerts a selective inhibition on certain subcortical structures and the autonomic nervous system. Chlorpromazine was found to differ from previously used sedatives and narcotics in producing calmness without significantly affecting clarity of consciousness. This novel property led to its designation as a tranquillizer.

As usually happens when an interesting and promising drug is discovered, research is directed into chemically related compounds in the hope of discovering more potent and therapeutically efficacious drugs. A large number of new phenothiazine derivatives have been introduced since the discovery of chlorpromazine, as shown in the Table.

\section{Chemical Constitution}

Chlorpromazine has stood the test of time and extensive clinical trials in all parts of the world. It is used as the standard for comparing the pharmacological properties of new phenothiazine derivatives.

Chlorpromazine and the other drugs shown in the Table have in common the phenothiazine nucleus, which consists of two benzene rings joined by a sulphur and a nitrogen atom as follows:

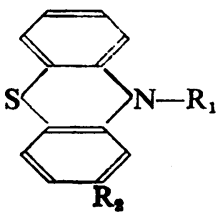

New derivatives are formed by substitution at positions $R_{1}$ and $\mathbf{R}_{2}$. The following are the most common substitutions at $\mathbf{R}_{1}$.

(1) A dimethylaminopropyl side chain found in chlorpromazine, promazine, acetylpromazine, and trifluopromazine.

(2) A piperidine side chain as in mepazine and thioridazine.

(3) A piperazine side chain as in prochlorperazine, perphenazine, trifluoperazine, and thiopropazate.

The substitutions at $\mathbf{R}_{2}$ include halogens, methoxy, acetyl, thiomethyl, and other organic radicals.

Differences in chemical structure of substituents are associated with differences in potency, pharmacological properties, clinical effects, side-effects, and toxic actions. Potency varies according to the type of radical at $\mathbf{R}_{\mathbf{2}}$. Chlorpromazine is more potent than promazine, and trifluopromazine is of greater potency still. Compounds with a piperazine side chain at $R_{1}$ are more potent than those with dimethylaminopropyl or piperidine side chains but have a greater tendency to produce extrapyramidal manifestations.

The drugs vary in their central sedative action. The piperazine derivatives tend to increase alertness and have greater anti-emetic action than chlorpromazine. Laevopromazine has greater antihistamine activity, hypotensive action, and more powerful potentiating action on central nervous system depressants than chlorpromazine and other drugs.

\section{Dosage}

The usual starting dose for adults is given in the Table. This is increased gradually until optimum clinical effects with minimum side-effects are achieved. Dosage has always to be determined individually, and the optimum dose will vary with the individual as well as the type and severity of the disorder. The maximum dosage shown in the Table is usually required only in severe psychiatric disorders.

\section{Side-effects and Toxic Reactions}

It is necessary to distinguish between side-effects on the one hand and toxic and hypersensitivity reactions on the other. Side-effects depend entirely on dosage and can occur in all patients if the dose is high enough. Toxic and hypersensitivity reactions occur only in some patients and are not closely correlated with dosage. Among the common side-effects are drowsiness and apathy, hypotension, dryness of mucous membranes, and extrapyramidal reactions.

Toxic and hypersensitivity reactions include, among others, dermatitis, blood dyscrasias, jaundice, and increased tendency to epileptic fits.

\section{Precautions}

Caution in prescribing these drugs is necessary if there is any evidence of depression of the bone-marrow activity. Blood examinations should be carried out at regular intervals during drug administration to detect the development of any blood dyscrasia, and a careful watch kept for relevant signs and symptoms. Caution is needed in administering these drugs to patients to whom hypotension constitutes a serious hazard-for example, those on the threshold of circulatory failure. In view of potentiating effects on other cerebral nervous system depressants, care is needed in prescribing with sedatives, narcotics, and anaesthetics.

\section{Chlorpromazine}

Chlorpromazine is a central-nervous-system depressant having little effect on the cerebral cortex but selective inhibiting actions on subcortical structures such as the reticular system, which subserves many functions, including degree of alertness; on the hypothalamus ; and 


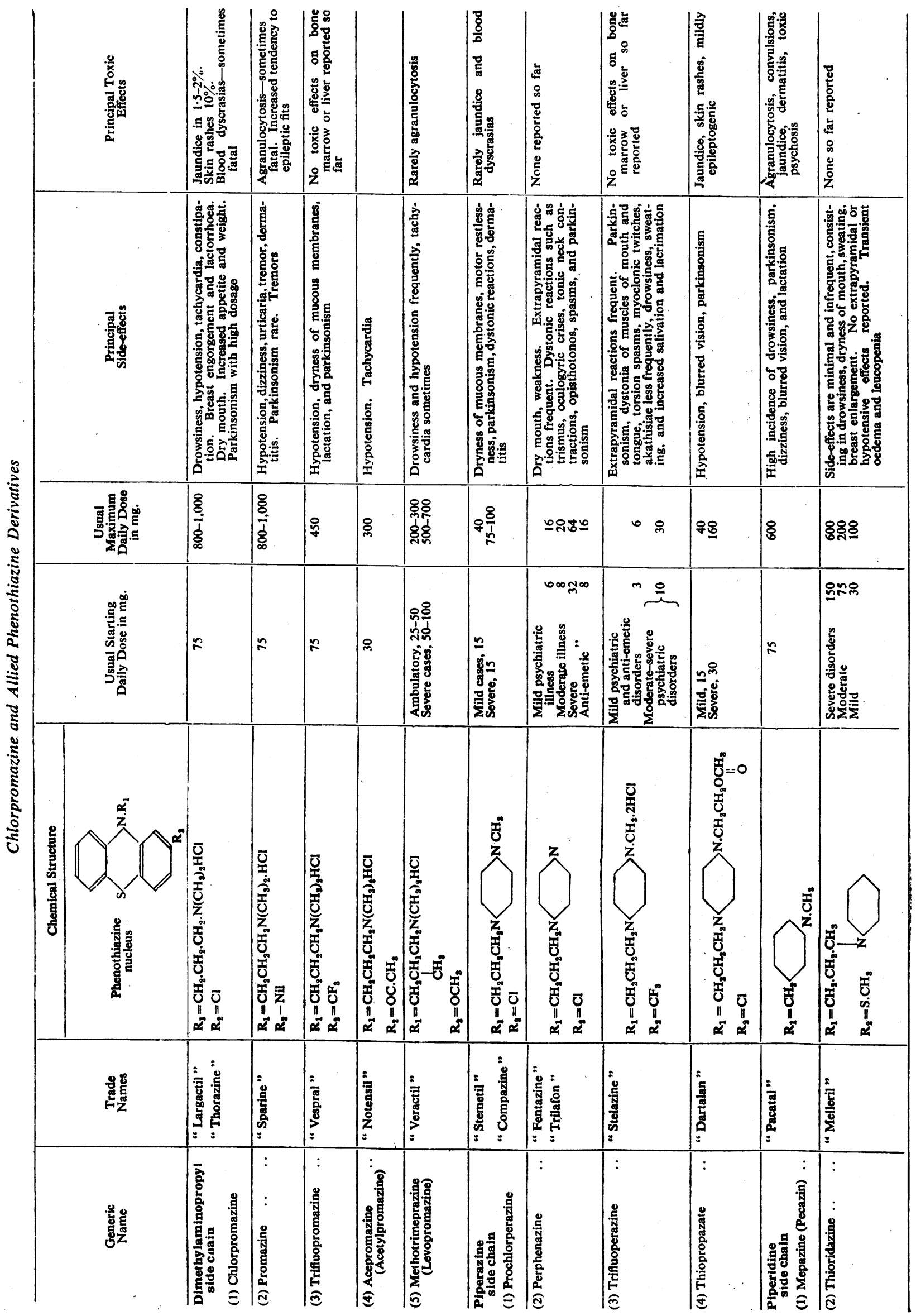


on various parts of the autonomic nervous system. Chemically it is anti-adrenergic, and this causes its hypotensive effects. It is also parasympatholytic, antiemetic, and hypothermic, and potentiates the effects of barbiturates, alcohol, and anaesthetics.

The following are the main therapeutic indications for chlorpromazine.

\section{General Medicine and Surgery}

Chlorpromazine is useful for relief of restlessness and agitation-for example, in patients with painful inoperable carcinoma, especially if complicated by persistent vomiting, or for restlessness and agitation in other physical disorders or associated with old age.

In low doses it has some value for relieving the vomiting of early pregnancy and the restlessness occurring in late pregnancy. Large doses should not be given just before or during labour, because of the risk of producing uterine inertia.

\section{Psychiatric Disorders}

(a) In acute and chronic schizophrenia for controlling disturbed behaviour and for relieving anxiety and agitation.

(b) For relieving anxiety, tension, agitation, and disturbed behaviour in other psychiatric illnesses including organic mental states. It is of particular value in the domiciliary management of patients in senile dementia exhibiting disturbed behaviour and restlessness.

(c) For the management of disturbed overactive behaviour in children and in mental defectives of all ages.

It has little or no therapeutic value in the treatment of depressive states, hysteria, or psychopathy, and is of only limited value in the treatment of anxiety states because it reduces energy and drive and because its side-effects often alarm patients.

\section{Promazine}

This drug differs chemically from chlorpromazine only in being without a chlorine atom at $\mathbf{R}_{2}$. Promazine is one-third as potent as chlorpromazine but is free from risk of producing jaundice and has a lower tendency to produce hypotension. It also has the practical advantage of being painless on intramuscular injection in contrast to chlorpromazine. However, promazine has disadvantages in tending to precipitate or increase the frequency of epileptic attacks; and in its tendency to produce agranulocytosis.

Its therapeutic indications are similar to those for chlorpromazine. It has no significant advantages except-in treating alcoholic disorders; its special value there is that it does not damage the liver, and helps symptomatically by its calming and anti-emetic effects. Because of its lesser tendency to lower blood-pressure it is valuable in treating elderly people, who are more prone to syncopal attacks due to hypotension. Thus promazine has a place in the treatment of mildly disturbed ambulant patients attending the general practitioner or out-patient clinic, and in the management of alcoholic disorders.

\section{Trifuopromazine}

This has similar clinical effects and therapeutic indications as chlorpromazine. Apart from producing its effects more quickly in lower dosage, it has no advantages over that drug.

\section{Acepromazine}

Acetyl promazine is twice as potent as chlorpromazine, but published reports provide little evidence that it is therapeutically valuable, and nowadays it is little used.

\section{Methotrimeprazine}

This drug, also called levopromazine, has similar clinical effects and pharmacological actions as chlorpromazine but is nearly twice as potent and helps some patients who fail to respond to chlorpromazine. It is a more potent antihistaminic than chlorpromazine. It has marked hypotensive effects necessitating care in its use in elderly patients and in patients on the threshold of circulatory failure. French authors have reported favourable results in depressive states, but the available evidence indicates that, though the drug may be effective for relieving anxiety and agitation associated with depressive illnesses, it does not materially benefit the underlying depression. It is also reported to be more effective than chlorpromazine for relieving intractable pain and associated emotional distress.

It appears to be only of limited value in schizophrenia for the treatment of neurosis.

\section{Pecazine}

This drug, also called mepazine, has twice as much sedative action as chlorpromazine and a greater parasympatholytic action. Its side-effects are more unpleasant than those of chlorpromazine, and published reports on its therapeutic efficacy are conflicting.

\section{Thiopropazate}

This has similar effects and therapeutic indication as chlorpromazine but is five times as potent and its clinical effects are produced more quickly. Further controlled trials are needed for its adequate assessment.

\section{Thioridaxine}

This is one of the most recently introduced phenothiazine derivatives and is chemically unique in having a thiomethyl radical at $\mathbf{R}_{2}$. Its tranquillizing properties are similar to those of chlorpromazine, but it differs in having little antiemetic action or potentiating effects on alcohol, barbiturates, and anaesthetics. The therapeutic indications are similar to those for chlorpromazine. The drug has been found effective for treating acute and chronic schizophrenics and for controlling disturbed overactive behaviour, marked anxiety, and agitation in other psychiatric disorders. It is much more selective in action than chlorpromazine, being almost a pure psychosedative. The exact assessment of its therapeutic value must await the results of fully controlled clinical trials.

\section{Prochlorperazine}

Prochlorperazine is a powerful anti-emetic, being four times as potent in this respect as chlorpromazine. It was first used clinically for treating nausea and vomiting, for prevention and treatment of attacks of migraine, and for the management of Ménière's syndrome and other forms of aural vertigo. As it had much lower sedative action on the central nervous system than chlorpromazine it was not, at first, used for treating psychiatric illnesses, but when later tried it was 
found to be effective for controlling overactive, aggressive, and disturbed behaviour in psychotic patients and to be superior to chlorpromazine for treating inert, withdrawn, and apathetic schizophrenics, in whom it had an altering effect, increasing both initiative and interest in the environment. Prochlorperazine is valuable for the management and treatment of alcoholism, as it relieves tension and anxiety, promotes natural sleep, and controls nausea and vomiting.

Side-effects are uncommon when the daily dose is below $40 \mathrm{mg}$. Above this dose restlessness and insomnia may occur, and with doses between 75 and $100 \mathrm{mg}$. extrapyramidal manifestations are frequent. These consist in Parkinsonian rigidity and sometimes the "excitomotor" syndrome-that is, stiffness of the jaws, followed by trismus, spasmodic protrusion of the tongue, lip tremors, speech difficulties, torticollis, conjugate eye deviation, choreoathetoid movements, excessive sweating, and tachycardia. These effects are readily abolished either by drugs that inhibit the symptoms of Parkinson's disease or by reduction in dosage.

\section{Perphenarine}

This drug is approximately five times as potent as chlorpromazine and has fewer side-effects. It does not produce drowsiness; on the contrary, some patients experience increased alertness and restlessness. Its main disadvantage is the tendency to cause extrapyramidal manifestations, particularly when the daily dosage exceeds $32 \mathrm{mg}$. These consist in dystonic disturbances such as trismus, oculogyric crises, and tonic neck contractions, which sometimes spread to the muscles of the back and produce opisthotonos. Parkinsonian rigidity sometimes occurs. These extrapyramidal effects can be controlled or abolished either by anti-Parkinsonian drugs or by reduction of dosage.

Perphenazine is effective in the treatment and management of acute schizophrenia and acute schizoaffective disorders, and for controlling excitement, anxiety, agitation, belligerence, and aggressive behaviour in chronic schizophrenia and other forms of chronic psychosis. The drug helps to relieve anxiety, tension, and depression in psychosomatic disorders and physical illnesses or disabilities. It is also indicated in cases of nausea and vomiting-for example, when these are associated with pregnancy, follow surgical operations or radiation therapy, are due to drugs, or have a psychogenic origin. Other uses are for the relief of intractable pain and associated emotional distress, and the treatment of drug addiction, particularly the management of withdrawal symptoms.

\section{Trifuoperaxine}

Trifluoperazine is about nine times more potent than chlorpromazine, having a more rapid onset of action and a more prolonged effect, which makes it unnecessary for administration to be more frequent than twice daily.

This drug helps to control disturbed behaviour, aggressiveness, hallucinations, and paranoid delusions in acute and chronic psychoses. It is more effective than chlorpromazine for treating withdrawn, apathetic, and inert schizophrenics, in whom it usually produces increased alertness, initiative, and energy.
In milder psychiatric disorders trifluoperazine is helpful for relieving anxiety, particularly when associated with listlessness and apathy, as it tends to restore initiative, increase energy, and improve concentration concomitantly with relief of anxiety.

\section{Conclusions}

The phenothiazine derivatives introduced after the discovery of chlorpromazine vary greatly in potency, therapeutic effects, and toxic actions.

Chlorpromazine has stood the test of extensive trial since it was first introduced nine years ago.

Promazine, though free from risk of producing jaundice, is less potent and has other serious toxic effects. It has a place in the treatment of alcoholic disorders and in patients in whom hypotension presents a serious hazard. The available evidence indicates that acetyl promazine and mepazine have no advantage over chlorpromazine and have more unpleasant side-effects. Laevopromazine is indicated when greater sedation is needed and is of some value for relieving anxiety and agitation in depressive states, though it does not appear to improve the underlying depression.

The piperazine phenothiazine substituents such as prochlorperazine, perphenazine, and trifluoperazine are more potent and less toxic than chlorpromazine but have a greater tendency to cause extrapyramidal reactions. They are more effective than chlorpromazine for treating inert, withdrawn schizophrenics. The recently introduced thioridazine is reported to be relatively free from side-effects and toxic actions and to be a pure psychosedative. Further fully controlled trials on thioridazine, trifluopromazine, and thiopropazate are necessary before their value and limitations can be assessed.

In conclusion we may say that the discovery of chlorpromazine and subsequent allied phenothiazine derivatives has opened new fields of therapeutics in medicine and surgery. In psychiatry phenothiazine derivatives have played an important part in the rapidly developing field of psychopharmacology, which is of great promise for advancing our knowledge of the causation as well as treatment of mental disorders.

REFERENCE: :

Laborit, H., and Huguenard, P. (1951). Presse méd., 59, 1329. $\longrightarrow$

\section{SOME PROBLEMS OF THE MEDICAL SERVICES OF THE FEDERATION OF RHODESIA AND NYASALAND}

\author{
BY
}

\author{
H. S. GEAR, M.D., D.P.H., D.T.M.\&H. \\ Consultant to the Federal Ministry of Health
}

That part of Africa now forming the Federation of the Rhodesias and Nyasaland has a recorded history of less than a hundred years. This history has greatly influenced the trend followed by the medical services of the Federation since they were formed in 1954, the year after the constitutional decisions were taken. When Livingstone explored the area it was the scene of slaveraiding, intertribal war, and uninhibited disease. Though the missionary societies soon set up their stations, little was altered until Cecil Rhodes turned his 